\title{
OLFACTORY DISORDERS IN SARS-COV-2 INFECTION: CONSIDERATIONS IN AGING
}

\author{
Alberto J. Mimenza-Alvarado ${ }^{1}$, José A. Avila-Funes ${ }^{1,2}$, and Sara G. Aguilar-Navarro ${ }^{1 *}$ \\ ${ }^{1}$ Department of Geriatric Medicine, Instituto Nacional de Ciencias Médicas y Nutrición Salvador Zubirán, Mexico City, \\ Mexico; ${ }^{2}$ University of Bordeaux, Inserm, Bordeaux Population Health Research Center, Bordeaux, France
}

\section{INTRODUCTION}

The COVID-19 pandemic is considered by the World Health Organization as a Public Health Emergency of International Concern. As of April 12, 2020, there were $1,696,588$ confirmed cases $(82,679$ new cases) of COVID-19 and 40,598 deaths (6,222 new deaths) worldwide. There is a global case fatality rate of $6.2 \%$. In the past 14 days, the number of new cases represents $65 \%(1,061,674$ cases $)$ of the total accumulated cases. To date, cases have been reported in 205 countries, in six WHO regions (America, Europe, Southeast Asia, Eastern Mediterranean, Western Pacific, and Africa). In Mexico, there are currently 4,661 confirmed cases and 296 deaths; 927 (19.9\%) of these correspond to adults over the age $60,53 \%$ males, and $48 \%$ females.

One of the defining characteristics of severe acute respiratory syndrome (SARS) - coronavirus (CoV)-19 is the predilection for infection in older adults and individuals with underlying chronic health conditions, resulting in higher incidents and mortality rates. Given that older adults experience a greater number and severity of chronic diseases and disabilities, as well as immune dysfunction, it is intuitive that COVID-19 will disproportionately affect this population. The aging process involves changes in various functions of the central and peripheral nervous system. One of them is the sense of smell. The changes that occur are the result of modifications at various levels: olfactory mucosa, nerve, and olfactory area, among others. As a result of recent reports of olfactory disorders in patients with SARS-CoV-2 infection (causative agent of COVID-19), reflection on its presence or absence as an early manifestation of COVID-19 among older adults is appropriate ${ }^{1}$. However, in this brief review, we would like to highlight the importance of the aging process and how it causes anatomical and pathophysiological changes specifically in the olfactory sense, and the importance of considering these changes when considering hyposmia as an early stage of COVID-19 infection in older adults.

\section{PHYSIOLOGY OF OLFACTORY DISORDERS IN AGING}

The prevalence of olfactory changes in older adults is $27 \%$ and, in general, it is more frequent in the elderly,
*Corresponding author:

Sara G. Aguilar-Navarro

E-mail: sara.aguilarn@incmnsz.mx
Received for publication: 14-04-2020

Approved for publication: 21-04-2020

DOI: 10.24875/RIC.20000157

0034-8376 / (c) 2020 Revista de Investigación Clínica. Published by Permanyer. This is an open access article under the CC BY-NC-ND license (http://creativecommons.org/licenses/by-nc-nd/4.0/). 
Figure 1. Special considerations for olfaction in older adults with severe acute respiratory syndrome (SARS)-Cov-2 infection. There are olfactory changes associated with aging. SARS-Cov-2 infection affects the olfactory bulb (pathway through which it enters the nervous system), which may worsen preexisting hyposmia in older adults, dashed line. Therefore, all these elements should be considered at the time of diagnosis.

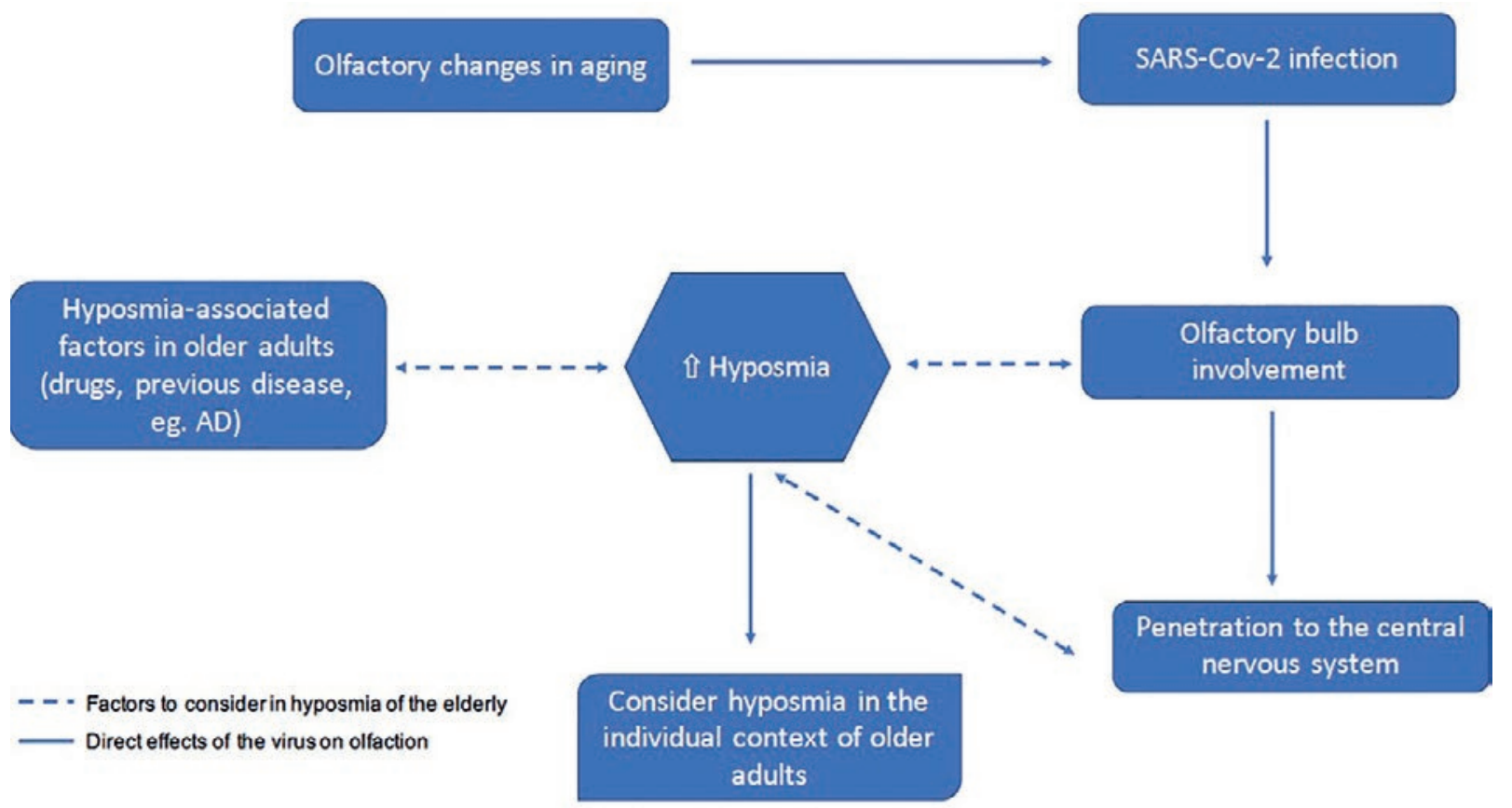

reaching $62.5 \%$ between the ages of 80 and 97 and it is considered more frequent among men². Changes in this regard include anatomical and physiological variables (decreased mucociliary clearance, atrophy of the nasal epithelium, decreased blood flow to the nasal mucosa, or decreased elasticity of mucus), as well as pathologies (chronic rhinosinusitis, nasal polyposis, etc.), which contribute to the higher prevalence. In the same way, the olfactory neuroepithelium also changes in its nature and integrity (decrease in the number of receptors, thinning of the epithelium, alterations in the cellular pattern, and in the distribution of the nucleus of the olfactory receptor, as well as the cells that support it).

The olfactory bulb and its laminae (mainly the glomerular layer) decrease with age. Furthermore, the volume of the hippocampus, amygdala, piriform cortex, anterior olfactory nucleus, and frontal pole of the brain (changes characteristically associated with cognitive disorders) decreases among older adults ${ }^{3}$. All the changes described above should be considered as they could explain the preexisting olfactory alterations in older adults. These changes could contribute to the harmful effect of SARS-CoV-2 on the olfactory system. Furthermore, it is necessary to consider other factors that increase the frequency of hyposmia associated with aging, which includes being a carrier of the APOE e4 genotype, atrial fibrillation, previous stroke and current smoking, and Alzheimer's disease (AD) ${ }^{4}$.

\section{COVID-19 AND EFFECT ON OLFACTION}

Studies conducted during the SARS epidemic, between 2002 and 2003, demonstrated the presence of SARS-CoV particles exclusively in neurons. Experimentally, inoculation in transgenic SARS-CoV or MERS-CoV mice has shown to penetrate intranasally into the brain, possibly through the olfactory nerve, spreading rapidly through the thalamus and brain stem, showing the potential mechanism of CoV neuroinvasion. Therefore, it is very likely, given the similarity of SARS-CoV-2, that it could share these neuroinvasive properties ${ }^{5}$. 
The recent retrospective study by Mao et al. showed that among 214 Wuhan individuals diagnosed with COVID-19, 78 patients ( $36.4 \%$ ) had symptoms in the nervous system: $53(24.8 \%)$ in the central nervous system (CNS), 19 (8.9\%) in the nervous system peripheral system, and 23 (10.7\%) in skeletal muscle mass. The most common neurological manifestations in the peripheral nervous system included hypogeusia $12(5.6 \%)$ and hyposmia 9 (5.1\%), although the most affected age group was not described ${ }^{6}$. The Italian study by Giacomeli et al. reported that among 69 patients diagnosed with COVID-19 (interquartile range of 50-74 years), 20 people (33.9\%) had taste or smell problems, and three patients $(5.1 \%)$ had marked hyposmia and no anosmia?.

At this time, it is important to consider hyposmia as a manifestation of SARS-CoV-2 (Covid-19), although caution should be taken when diagnosing older adults, since the olfaction system is affected during aging. In addition, hyposmia is associated with neurodegenerative diseases (e.g., AD), and with the use of drugs (angiotensin-converting enzyme inhibitors [ACE-inhibitors] and antidepressants), thus, the absence of this symptom could mask the diagnostic role of olfactory alteration in acute SARS-CoV2 infection (Fig. 1).

The current recommendation is to accurately locate the temporality of the symptom, even ruling out the presence of associated taste alteration. In this way, the presence of this alteration will be better described in future studies and will therefore allow us to define the frequency, severity, and reversibility of this interesting symptom associated with COVID-19 infection.

Furthermore, caution should be given when assessing this symptom in older adults. At present, the consequences that the SARS-CoV-2 invasion could cause on CNS and its role in neuronal function (particularly cognitive function) are unknown. However, it is necessary to keep in mind that olfactory changes in older adults have also been associated with other neurodegenerative diseases linked with viruses.

\section{REFERENCES}

1. D’Adamo H, Yoshikawa T, Ouslander JG. Coronavirus disease 2019 in geriatrics and long-term care: the ABCDs of COVID-19. J Am Geriatr Soc. 2020;2020:16445.

2. Murphy C, Schubert CR, Cruickshanks KJ, Klein BE, Klein R, Nondahl DM. Prevalence of olfactory impairment in older adults. JAMA. 2002;288:2307-12.

3. Doty RL, Kamath $\mathrm{V}$. The influences of age on olfaction: a review. Front Psychol. 2014;5:20.

4. Palmquist E, Larsson $M$, Olofsson JK, Seubert J, Bäckman L, Laukka EJ. A Prospective study on risk factors for olfactory dysfunction in aging. J Gerontol A Biol Sci Med Sci. 2020;75:603-10.

5. Li YC, Bai WZ, Hashikawa T. The neuroinvasive potential of SARS-CoV2 may play a role in the respiratory failure of COVID-19 patients. J Med Virol. 2020;2020:25728.

6. Mao L, Jin H, Wang M, Hu Y, Chen S, He Q, et al. Neurologic manifestations of hospitalized patients with coronavirus disease 2019 in Wuhan, China. JAMA Neurol. 2020;2020:e201127.

7. Giacomelli A, Pezzati L, Conti F, Bernacchia D, Siano M, Oreni L, et al. Self-reported olfactory and taste disorders in SARS-CoV-2 patients: a cross-sectional study. Clin Infect Dis. 2020;26: ciaa330. 